\title{
NGC 3310, a galaxy merger?
}

\author{
M. Kregel ${ }^{1}$ and R. Sancisi ${ }^{1,2}$ \\ 1 Kapteyn Astronomical Institute, University of Groningen, PO Box 800, 9700 AV Groningen, The Netherlands \\ 2 Osservatorio Astronomico, Bologna, Italy \\ Received 20 March 2001 / Accepted 18 June 2001

\begin{abstract}
The H I structure and kinematics of the peculiar starburst galaxy NGC 3310 (Arp 217, UGC 5786) are discussed. New evidence bearing on the origin of the starburst is presented. The bulk of H I coincides with the bright optical disk and shows differential rotation. Its velocity dispersion is, however, unusually large for a spiral galaxy ( up to $\simeq 40 \mathrm{~km} \mathrm{~s}^{-1}$ ), suggesting that the disk is highly perturbed as already indicated by optical emission somewhat patchy, to the south. These H i tails, the perturbed kinematics and the peculiar optical morphology strongly suggest a recent merger between two gas-rich galaxies. This seems to have been a major merger in which most of the gas in the inner parts has been preserved in neutral atomic form and either one of the progenitor disks has survived or a new disk has formed.
\end{abstract} \\ line spectroscopy. There are, in addition, two prominent $\mathrm{H}_{\mathrm{I}}$ tails, one extending to the north-west and the other,
}

Key words. galaxies: individual: NGC 3310 - galaxies: starburst - galaxies: interactions - galaxies: kinematics and dynamics - radio lines: galaxies

\section{Introduction}

The galaxy NGC 3310, located in the vicinity of the Ursa Major cluster, lies at a distance of $\sim 13 \mathrm{Mpc}^{1}$ and is classified as SABbc(r)pec (de Vaucouleurs et al. 1991). Van der Kruit \& de Bruyn (1976) have investigated its group membership and its environment and have concluded that it has no companions. Its main global properties are listed in Table 1. NGC 3310 is a relatively small system undergoing a strong starburst (Telesco \& Gatley 1984; Smith et al. 1996). The optical morphology is illustrated in Fig. 1. The bright inner region is dominated by a two-armed open spiral pattern in $\mathrm{H} \alpha$ (van der Kruit \& de Bruyn 1976; Balick \& Heckman 1981; Mulder \& van Driel 1996). The inner part of this well-developed pattern connects to a $\sim 900 \mathrm{pc}$ diameter starburst ring, surrounding the blue compact nucleus. The circumnuclear regions show a moderately low metallicity, whereas the nucleus has solar abundances (Pastoriza et al. 1993). The Far UV and $B$-band surface brightness profiles of NGC 3310 are very similar and seem to follow an $R^{1 / 4}$ law outside the inner starburst ring (Smith et al. 1996). The outer parts of NGC 3310 are dominated by the so-called "bow-andarrow" structure (Walker \& Chincarini 1967). The diffuse "bow" at the western side is a ripple (at $\sim 6 \mathrm{kpc}$ from the center) consisting primarily of late-type stars, possibly

\footnotetext{
Send offprint requests to: M. Kregel,

e-mail: kregel@astro.rug.nl

1 All distance dependent parameters are calculated using $H_{0}=75 \mathrm{~km} \mathrm{~s}^{-1} \mathrm{Mpc}^{-1}$.
}

debris from an accreted disk (Schweizer \& Seitzer 1988). A second fainter ripple can be seen farther out to the north-west. The "arrow" (extending from 4 to $9 \mathrm{kpc}$ from the center to the north-west) is a chain of bright knots consisting of stellar clusters most likely containing young massive stars and has a similar age as the central starburst ring (Smith et al. 1996). The "bow-and-arrow" structure has been interpreted as a one-sided jet emanating from the nucleus (Bertola \& Sharp 1984) or as the result of the accretion of a small gas-rich galaxy (Balick \& Heckman 1981; Schweizer \& Seitzer 1988; Mulder et al. 1995; Smith et al. 1996). Besides the "arrow", other knots with recent star formation are seen at the northern and southern part of the disk (van der Kruit \& de Bruyn 1976, their Fig. 1).

The ionized gas shows large streaming motions (up to $60 \mathrm{~km} \mathrm{~s}^{-1}$ ) along the arms (van der Kruit 1976; Grothues \& Schmidt-Kaler 1991), which are consistent with the presence of a strong density wave (van der Kruit 1976; Mulder \& van Driel 1996). The $\mathrm{H} \alpha$ rotation curve in the nuclear region is one of the steepest rising rotation curves found in spiral galaxies (van der Kruit 1976; Grothues \& Schmidt-Kaler 1991). Another peculiarity is the offset between the dynamical center and the stellar nucleus of $(96 \pm 19)$ pc (Walker \& Chincarini 1967; van der Kruit 1976; Balick \& Heckman 1981).

Radio continuum maps (van der Kruit \& de Bruyn 1976; Balick \& Heckman 1981; Duric et al. 1986) show extended, remarkably bright synchotron emission from the inner regions and strong sources coincident with the giant $\mathrm{H}$ II regions in the arms, in the inner ring, and in the 
Table 1. Properties of NGC 3310.

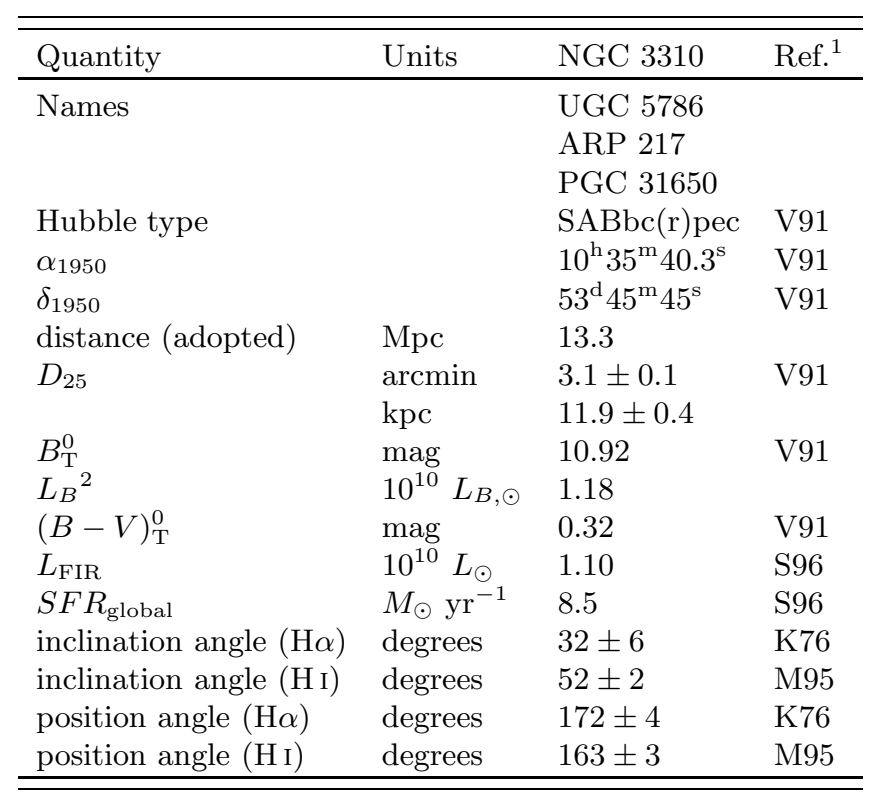

1. Acronyms; K76 - van der Kruit (1976), V91 de Vaucouleurs et al. (1991), M95 - Mulder et al. (1995) and S96 - Smith et al. (1996).

2. The $B$-band magnitude is converted to luminosity in solar units using $M_{B, \odot}=5.48$.

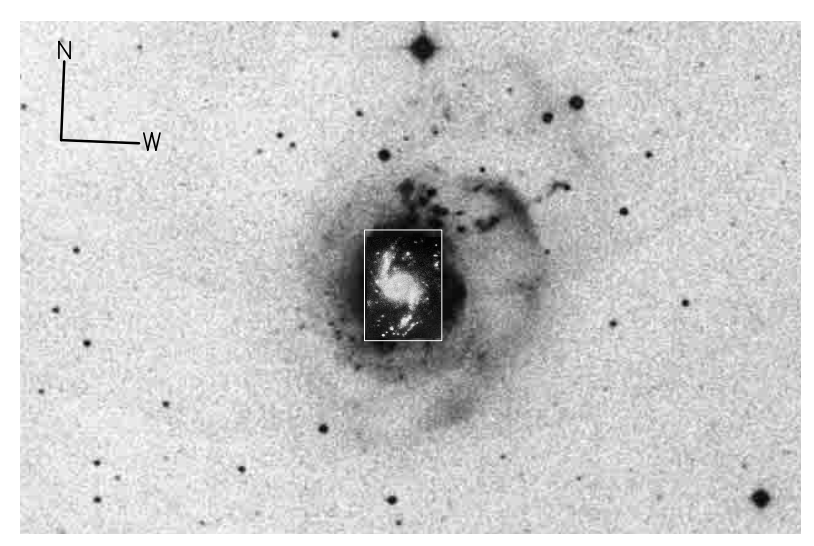

Fig. 1. An $\mathrm{H} \alpha$ image (taken by H.C. Arp) overlayed on a negative optical $B$-band image (both images are from van der Kruit $\&$ de Bruyn 1976). The length of the $\mathrm{N}-\mathrm{W}$ indicators is $1 \operatorname{arcmin}(=3.9 \mathrm{kpc})$.

nucleus. The strong IR emission correlates well with both the continuum and $\mathrm{H} \alpha$ emission (Telesco \& Gatley 1984).

An earlier study of the neutral hydrogen (Mulder et al. 1995) revealed an H I extension of the optical "arrow" out to $\sim 24 \mathrm{kpc}$ and extended $\mathrm{HI}$ at $\sim 37 \mathrm{kpc}$ to the south of the nucleus with no optical counterpart. Another peculiarity was a "hole" in the H I distribution centered on the south-eastern part of the optical ring (SN 1991N is situated near the center of this "hole"). The molecular gas shows a clumpy distribution and is primarily associated with the spiral arms (Kikumoto et al. 1993; Mulder et al. 1995). In the nucleus it is less clear: a small amount
Table 2. WSRT Observing Parameters.

\begin{tabular}{|c|c|c|}
\hline Observation & $\mathrm{A}$ & \\
\hline $12 \mathrm{hrs}, 36 \mathrm{~m}$ & & 16 July 1997 \\
\hline $12 \mathrm{hrs}, 72 \mathrm{~m}$ & & 11 June 1997 \\
\hline & $\mathrm{B}$ & \\
\hline $12 \mathrm{hrs}, 72 \mathrm{~m}$ & & 15 Jan. 1987 \\
\hline Field centers & $\mathrm{A}$ & \\
\hline$\alpha_{1950}$ & & $10^{\mathrm{h}} 35^{\mathrm{m}} 40.00^{\mathrm{s}}$ \\
\hline$\delta_{1950}$ & & $53^{\mathrm{d}} 43^{\mathrm{m}} 0.01^{\mathrm{s}}$ \\
\hline & $\mathrm{B}$ & \\
\hline$\alpha_{1950}$ & & $10^{\mathrm{h}} 35^{\mathrm{m}} 40.1^{\mathrm{s}}$ \\
\hline$\delta_{1950}$ & & $53^{\mathrm{d}} 45^{\mathrm{m}} 49^{\mathrm{s}}$ \\
\hline \multirow[t]{2}{*}{ Central velocity $\left(\mathrm{km} \mathrm{s}^{-1}\right)$} & $\mathrm{A}$ & 970.00 \\
\hline & $\mathrm{B}$ & 1000.00 \\
\hline \multirow{2}{*}{ Baselines (m) } & $\mathrm{A}$ & $36: 2736: 36$ \\
\hline & $\mathrm{B}$ & $36: 2700: 72$ \\
\hline Bandwidth (MHz) & & 5 \\
\hline Number of channels & & 63 \\
\hline Channel separation $\left(\mathrm{km} \mathrm{s}^{-1}\right)$ & & 16.6 \\
\hline \multirow[t]{2}{*}{ Velocity weighting } & $\mathrm{A}$ & Uniform \\
\hline & $\mathrm{B}$ & Hanning \\
\hline Synthesized beam (FWHM) & $\mathrm{C}$ & $14^{\prime \prime} .2 \times 17^{\prime \prime} .7$ \\
\hline Velocity resolution $\left(\mathrm{km} \mathrm{s}^{-1}\right)(\mathrm{FWHM})$ & $\mathrm{C}$ & 33.3 \\
\hline Noise level $(1 \sigma)\left(\mathrm{mJy}\right.$ beam $\left.^{-1}\right)$ & $\mathrm{C}$ & 0.8 \\
\hline$(\mathrm{K})$ & & 1.92 \\
\hline
\end{tabular}

of molecular gas is not ruled out. The total $\mathrm{H}_{2}$ mass, obtained using the Galactic $\mathrm{CO}-\mathrm{H}_{2}$ conversion factor, is $\sim 2 \times 10^{8} M_{\odot}$ (Kikumoto et al. 1993; Mulder et al. 1995), a value typical for late-type galaxies.

In summary, many features of NGC 3310 - the unusual morphology, the starburst features, the streaming of ionized gas along the arms, the offset between the dynamical center and the nucleus, the ripples in the outer parts, the "arrow" and its H I counterpart - indicate that some major disturbance has affected gas and stars and has led to massive star formation. These features together with the Far UV and $B$-band $R^{1 / 4}$ surface brightness profiles indicate that NGC 3310 may well be the result of a merger event.

New H I data, obtained with the Westerbork Synthesis Radio Telescope (WSRT) and combined with those of Mulder et al. (1995) are presented here. They give additional information concerning the origin of the starburst and of the optical and $\mathrm{H}$ I peculiarities.

\section{Observations and data reduction}

The new 21-cm line observations of NGC 3310 were obtained with the WSRT as a part of WHISP (Westerbork H I Survey of Spiral and Irregular Galaxies) (Swaters et al. 2001). Due to telescope maintainance only 27 interferometers were available during these observations.

The main observational parameters are listed in Table 2, where "A" denotes the WHISP observations, "B" the observations obtained by Mulder et al. (1995) and "C" the combination of "A" and "B" (described below). 


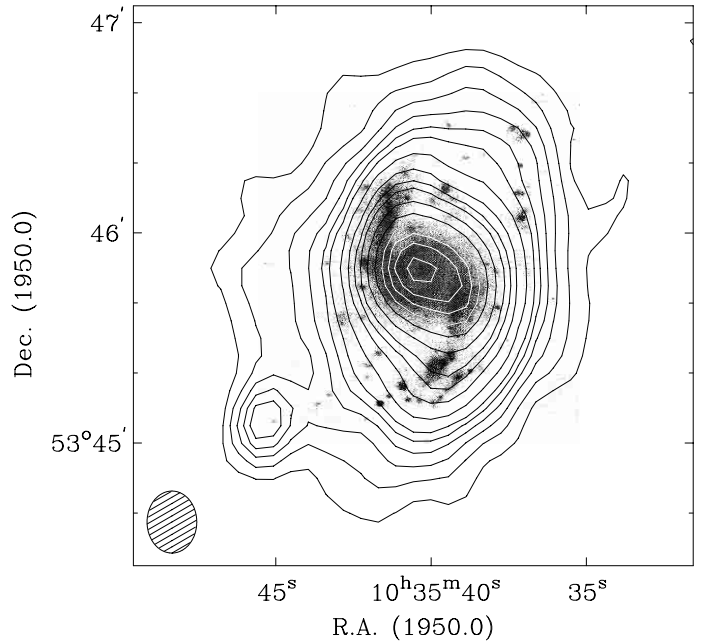

Fig. 2. Full resolution $\left(14^{\prime \prime} .2 \times 17^{\prime \prime} .7\right)$ map of the radio continuum emission from NGC 3310 at $1.42 \mathrm{GHz}$. The rms noise is $0.29 \mathrm{mJy} \mathrm{beam}^{-1}$, contour levels are (first:last:increment) 1:6:1, 8:17:3, 23:51:7 and $59 \mathrm{mJy}$ beam $^{-1}$. The $\mathrm{H} \alpha$ image (van der Kruit \& de Bruyn 1976) is shown in greyscale.

The combined observations were smoothed to $299^{\prime \prime} 9 \times 30^{\prime \prime} .7$ and $60^{\prime \prime} 9 \times 63^{\prime \prime} .0$ to study the extended, low surface brightness structures.

The map of the radio continuum was obtained from a linear fit of a baseline over the line free channels on either side of the band (28 channels in total) and was subsequently subtracted from the datacube. The resulting line maps were cleaned to correct for instrumental effects like sidelobes, grating rings and offset baselevels. The search area was determined for each channel map separately, in some cases after smoothing to $90^{\prime \prime}$ to identify the regions containing extended emission. After cleaning, the new data and those of Mulder et al. (1995) were combined.

The H I channel maps are shown in Fig. 4. The contours show the distribution of $\mathrm{H}$ I intensity at $30^{\prime \prime}$ resolution. The extended emission is shown at $60^{\prime \prime}$ resolution by the shaded areas. The optical picture and the "dirty" $30^{\text {" }}$ radio continuum map are also shown.

\section{Analysis}

\subsection{Radio continuum}

Figure 2 shows the cleaned radio continuum map of NGC 3310 at full resolution. The extended source of emission includes nucleus, central ring and spiral arms; its peak surface brightness is $63 \mathrm{mJy}_{\text {beam }}{ }^{-1}$. The $5.5 \mathrm{mJy}_{\text {beam }}{ }^{-1}$ point source, visible $\sim 1^{\prime}$ south-east of the nucleus, is probably a background source. The position of the nucleus cannot be determined accurately for such an extended source. The value of the continuum flux at $1.42 \mathrm{GHz}$ (Table 3) agrees with those published by Lequeux (1971) and by Duric et al. (1986). The total power is $21.85 \pm 0.03$ $\left(\log \left(P_{1.42 \mathrm{GHz}} / \mathrm{W} \mathrm{Hz}^{-1}\right)\right)$.

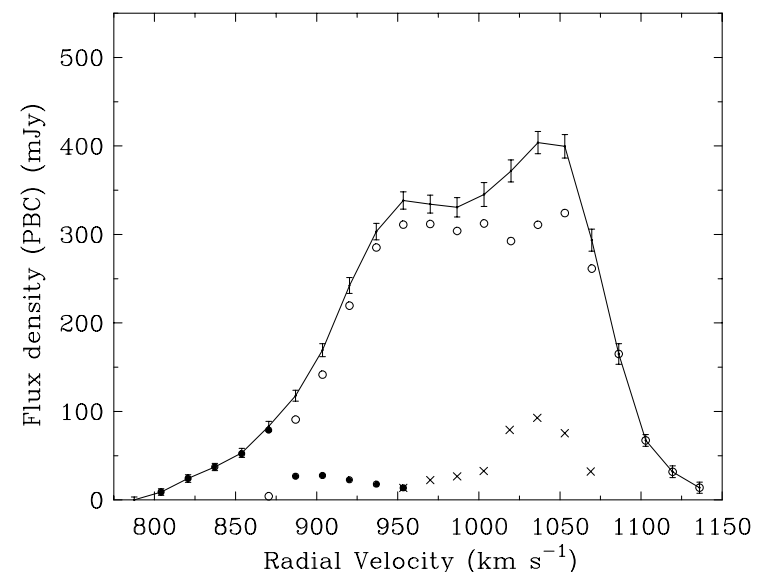

Fig. 3. Global $21 \mathrm{~cm}$ line profile of NGC 3310 (solid line). The dots show the northern tail, the crosses the southern tail and the circles the $\mathrm{H}$ I disk.

Table 3. Integral H i properties and the radio continuum flux of NGC 3310.

\begin{tabular}{lll}
\hline \hline Quantity & Units & Value \\
\hline $\int S \mathrm{~d} v$ & $\mathrm{Jy} \mathrm{km} \mathrm{s}$ & $69 \pm 4$ \\
$M_{\mathrm{H} \mathrm{I}}$ & $10^{9} M_{\odot}$ & $2.8 \pm 0.2$ \\
$S_{1.42 \mathrm{GHz}}$ & $\mathrm{Jy}$ & $0.34 \pm 0.01$ \\
\hline \hline
\end{tabular}

\subsection{Anomalous $\mathrm{HI}$}

The H I line flux was determined in each channel map at $60^{\prime \prime}$ resolution. The resulting global $\mathrm{H}$ I profile is shown in Fig. 3. It is similar to that obtained by Mulder et al. (1995). The asymmetric structure and the remarkable tail on the low velocity side clearly point at some anomaly in the $\mathrm{H}$ I distribution and/or kinematics. The integral quantities derived from the global profile are listed in Table 3.

The channel maps (Fig. 4) show that the bulk of $\mathrm{H} \mathrm{I}$ coincides with the bright optical disk and has the characteristic pattern of differential rotation. There are, in addition, two extended features, one on the north-west side visible in the velocity range 870 to $953 \mathrm{kms}^{-1}$ and the other to the south, clearly visible (shaded) at velocities 1003 to $1069 \mathrm{~km} \mathrm{~s}^{-1}$. Both have already been noticed and reported by Mulder et al. (1995). The better sensitivity of the data presented here makes it possible, however, to significantly improve the study of their structure and kinematics. In the following, these two extended features will be referred to as the northern and the southern tail.

\section{a) Northern tail}

The H I extension to the north-west (Fig. 4) coincides with the already mentioned and well-known "arrow". One cannot fail to notice the continuity in space and velocity of this feature with the emission in the channel maps at lower velocities, from 854 to $804 \mathrm{~km} \mathrm{~s}^{-1}$. It should also be noted that these are precisely the velocities of the anomalous tail in the global H I profile. It is therefore natural to associate 


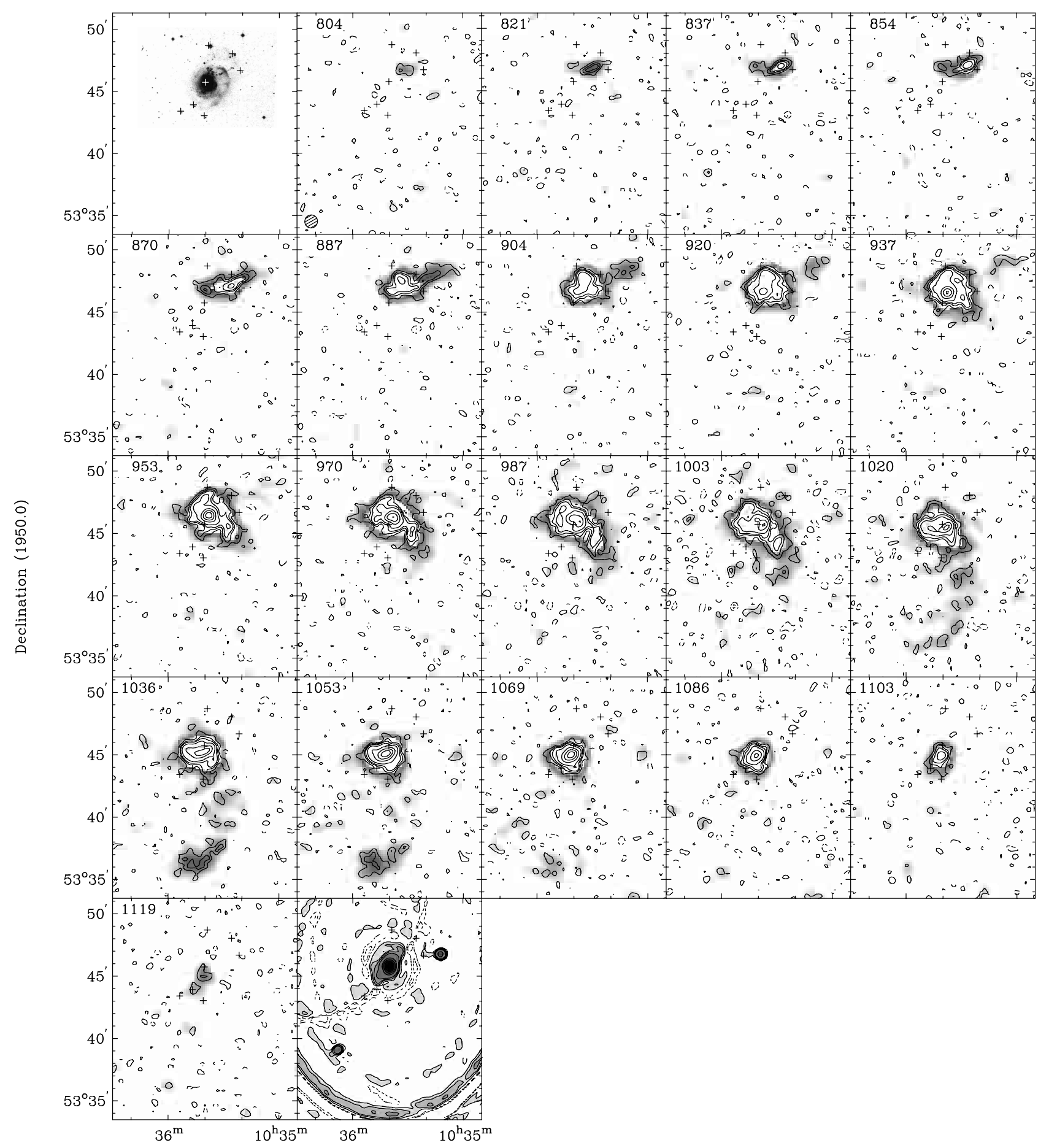

Right Ascension (1950.0)

Fig. 4. H I channel maps of NGC 3310. The beam size (HPBW) is $29^{\prime \prime} 9 \times 30^{\prime \prime} .7$. The contour levels are $-3.2,-1.6,1.6(\sim 2 \times$ rms noise), $3.2,4.8,6.3,7.8,15.7,23.6$ and $31.4 \mathrm{mJy}_{\text {beam }}{ }^{-1}$. The shading shows the faint extended emission in the outer parts on a logarithmic scale up to $6.5 \mathrm{mJy}^{\text {beam }}{ }^{-1}$ at a resolution of $60^{\prime \prime} .9 \times 63^{\prime \prime} .0$. Also the optical image (top left) and the radio continuum image used for subtraction (bottom right) are shown. The radial velocities (in $\mathrm{km} \mathrm{s}^{-1}$ ) are heliocentric.

the $\mathrm{HI}$ in the anomalous $804-854 \mathrm{~km} \mathrm{~s}^{-1}$ velocity range with the anomalous $\mathrm{H}$ I pointing away from the main body (the "arrow") in the adjacent, higher velocity channels 870 to $953 \mathrm{~km} \mathrm{~s}^{-1}$. The resulting structure has the shape of a tail (see Fig. 5) located on the northern edge of the disk, slightly curved and elongated from the east to the north-west. It has been obtained by adding the Hi over the whole velocity range 804 to $953 \mathrm{~km} \mathrm{~s}^{-1}$ and by taking, at velocities 887 to $953 \mathrm{~km} \mathrm{~s}^{-1}$, only the emission from the "arrow". There is a striking coincidence of this H I tail not only with the optically bright knots of the "arrow" itself but also with the bright compact knots seen along a 


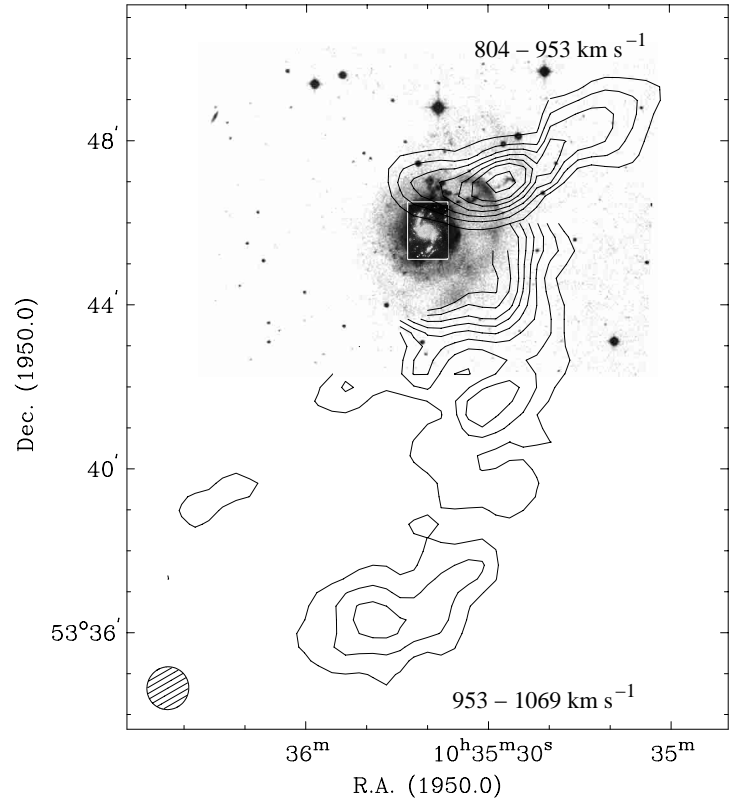

Fig. 5. Map showing the H I tails of NGC 3310 overlayed on the optical picture. The beam size is $60 .{ }^{\prime \prime} 9 \times 63^{\prime \prime} .0$. The contour levels are $0.4,0.8,1.2,1.6,2.0,2.4,3.0$ and $3.6 \times 10^{20} \mathrm{~cm}^{-2}$. The velocity ranges for the northern and the southern tail are indicated.

curve on the northern side of the bright disk of NGC 3310. Since the tail is unresolved at $60^{\prime \prime}$, the same procedure as described above was repeated at $30^{\prime \prime}$ and the result is shown in Fig. 6a. The coincidence with the optical knots is even clearer, suggesting a physical association of the knots with the $\mathrm{H}$ I tail. These knots should then have the same velocity as the $\mathrm{HI}$; but according to the $\mathrm{H} \alpha$ data published by Mulder \& van Driel (1996), their velocities seem to be around $950 \mathrm{~km} \mathrm{~s}^{-1}$, difficult to reconcile with the velocity of the $\mathrm{HI}$ indicated here.

The kinematics and structure of the tail are illustrated in the position-velocity map of Fig. 6b which was constructed along the path marked by the crosses in Fig. 6a. The distance along the tail in Fig. 6b is measured with respect to the most eastern marker. The figure shows the $\mathrm{H}$ I of the disk, which is centered at $950 \mathrm{~km} \mathrm{~s}^{-1}$, and the tail (shaded), which is in the velocity range 800 to $940 \mathrm{~km} \mathrm{~s}^{-1}$. There is a dip in the tail at a distance of $150^{\prime \prime}$ and a velocity of $850 \mathrm{~km} \mathrm{~s}^{-1}$. The main properties of the tail are listed in Table 4 . Its H I mass is about $8 \%$ of the total hydrogen mass of NGC 3310.

Clearly, if the above analysis is valid and it is correct to connect the kinematically anomalous $\mathrm{H}$ I with that of the "arrow" as we have done, a completely new physical picture emerges: instead of a straight jet pointing outward from the nucleus as suggested before (Bertola \& Sharp 1984), we have a curved tail-like structure attached to the northern edge of the bright optical disk.

\section{b) Southern tail}

The channel maps in Fig. 4 show an extended, peculiar feature (shaded area) on the southern side of NGC 3310
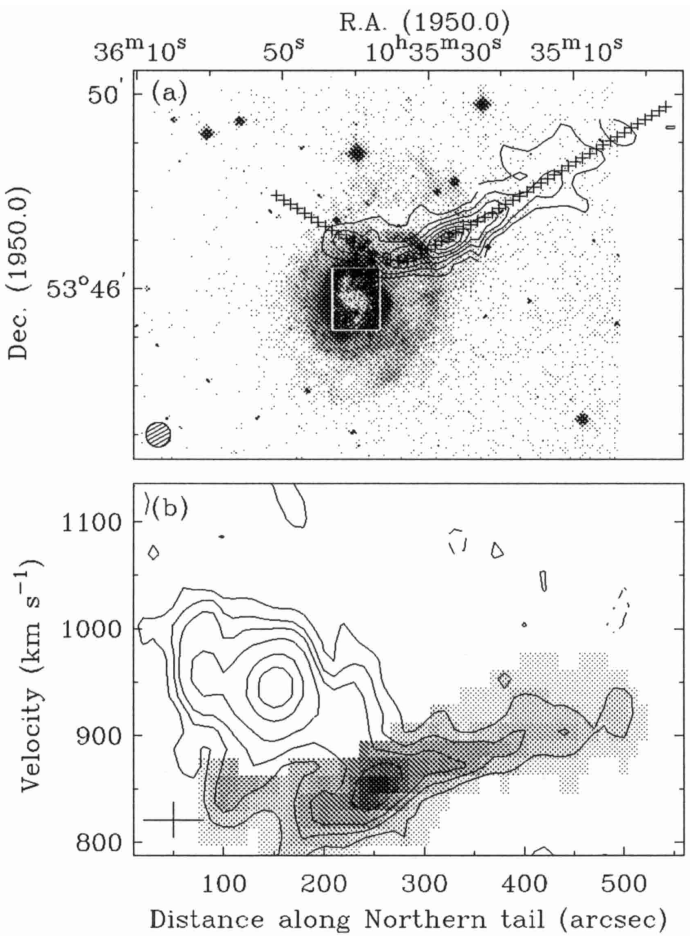

Fig. 6. a) $30^{\prime \prime}$ contourmap of the northern tail overlayed on the optical picture. The contour levels are 1, 2, 3, 4, 5, 6 and $\left.7 \times 10^{20} \mathrm{~cm}^{-2}, \mathbf{b}\right)$ position-velocity map along the track from east to west marked in a) following the ridge of the northern tail. The contour levels are as for the channel maps (Fig. 4). In this position-velocity diagram the tail is shown shaded.

Table 4. H I properties of the tails of NGC 3310.

\begin{tabular}{llll}
\hline \hline Quantity & Units & Northern & Southern \\
\hline$M_{\mathrm{H}}$ & $10^{8} M_{\odot}$ & $2.3 \pm 0.2$ & $2.7 \pm 0.2$ \\
Length & $\mathrm{kpc}$ & 23 & 51 \\
$\left(N_{\mathrm{H}}\right)_{\max }$ & $10^{20} \mathrm{~cm}^{-2}$ & 7.5 & $\sim 1.5$ \\
$\sigma_{\mathrm{H}}$ & $\mathrm{km} \mathrm{s}^{-1}$ & 17 & 13 \\
\hline \hline
\end{tabular}

at radial velocities ranging from 953 up to $1069 \mathrm{kms}^{-1}$. It has a somewhat patchy, but coherent tail-like structure (see also Fig. 5) connecting smoothly to the H I disk on its western side. The southern structure shown in Fig. 5 was obtained by adding all channel maps in the above velocity range.

Figure 7a shows the tail and the total $\mathrm{H}$ I at $60^{\prime \prime}$ resolution. The crosses mark the path along which the positionvelocity map shown in Fig. 7b was constructed. The distance along the tail in Fig. $7 \mathrm{~b}$ is with respect to the most northern marker. The figure shows a clear continuity in both space and velocity from the inner to the outer regions. The tail seems to be an extension of the western part of the $\mathrm{H}$ I disk (the emission closer to the bright disk has been omitted because of confusion). Its main properties are given in Table 4 . Its $\mathrm{H}$ I mass is at least $9 \%$ of the total hydrogen mass of NGC 3310.

In addition to the two well-developed H I tails, Fig. 5 also shows hints of an isolated feature to the south-east of 

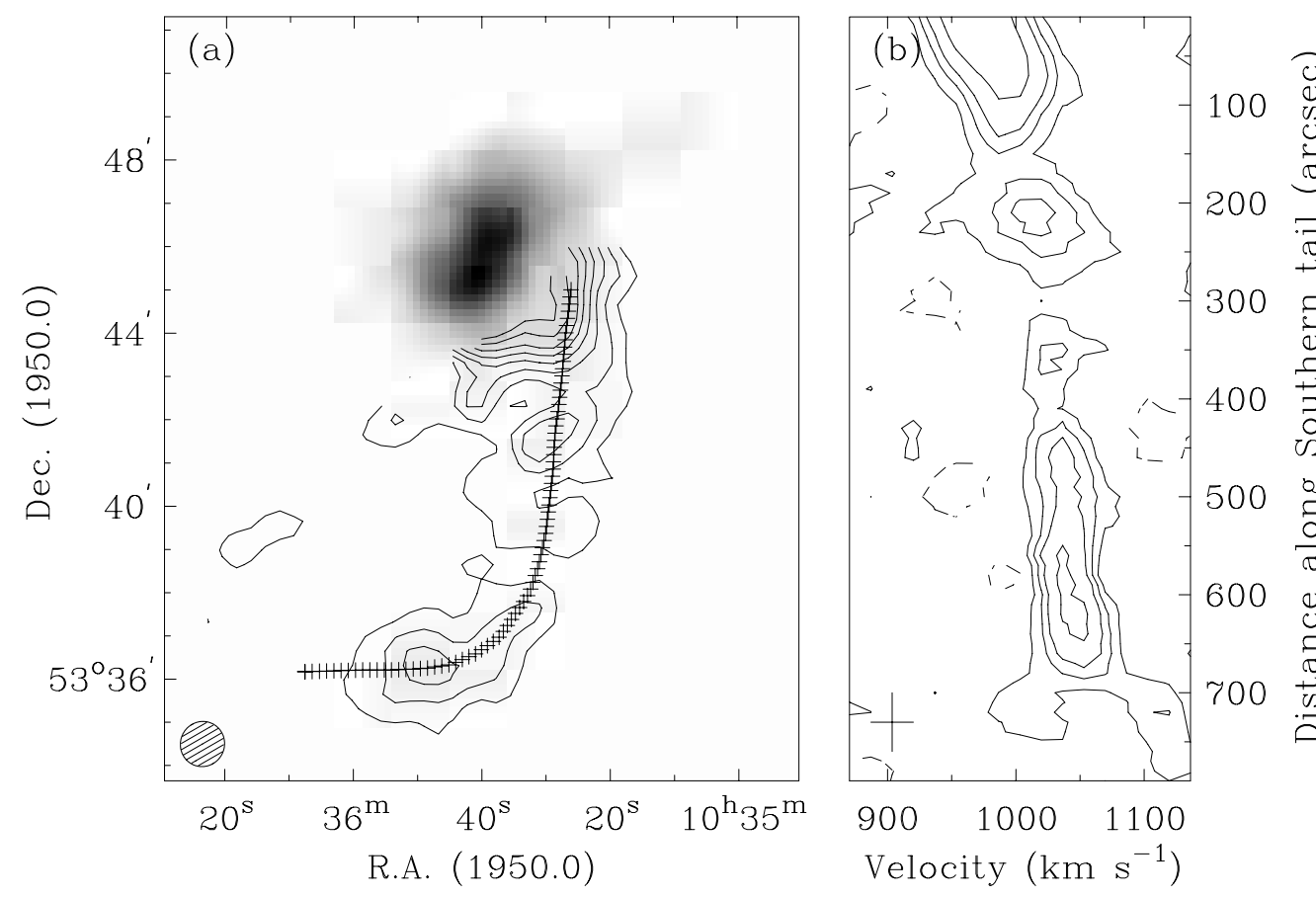

Fig. 7. a) The southern $\mathrm{H}$ I tail is represented in contours (same as in Fig. 5). The shading shows the total H I density distribution of NGC 3310 at $60^{\prime \prime}$ resolution (obtained by integrating over all velocities), b) position-velocity map along the track following the ridge of the tail from north to south, as marked in a). The contour levels are $-2.1,2.1,4.3,6.4$, and $10.7 \mathrm{mJy}^{\mathrm{beam}}{ }^{-1}$.

NGC 3310, visible (faint shaded areas) in Fig. 4 at velocities of 1053 and $1069 \mathrm{~km} \mathrm{~s}^{-1}$. This could be part of the southern tail described above. The present observations, however, are not sensitive enough to be sure of the reality of this feature.

\subsection{HI disk}

The overall $\mathrm{H}$ I density distribution and the velocity field in and around NGC 3310 are shown in Figs. 9a and b. More detailed maps of the $\mathrm{H}$ I disk, at 20 arcsec resolution, are presented in Figs. 9c and d. The high-resolution total H I map was obtained by defining the area of the emission for each channel map using the lower-resolution maps as masks. The velocity field was obtained by taking the density weighted mean with a $1.7 \mathrm{mJy}^{\text {beam }}{ }^{-1}$ $(2 \sigma)$ cutoff. In the construction of these maps, the emission from the northern and southern tails, clearly visible in the $60^{\prime \prime}$ channel maps, was masked out in order to have a clearer picture of the disk structure and kinematics.

In the $60^{\prime \prime}$ map, the central surface density reaches $\sim 2.4 \times 10^{21} \mathrm{~cm}^{-2}$ which corresponds to $\sim 11 M_{\odot} \mathrm{pc}^{-2}$ in the plane of the galaxy. This is similar to the values found for normal high surface brightness galaxies (Broeils $\&$ van Woerden 1994; Rhee \& van Albada 1996). The 20" resolution map shows that the peak surface density is actually $\sim 4.5 \times 10^{21} \mathrm{~cm}^{-2}$ and is reached about $30^{\prime \prime}$ south of the optical center.

The $20^{\prime \prime}$ map (Fig. 9c) shows a disk with a bright inner part and a large depression centered $\sim 8^{\prime \prime}(\sim 500 \mathrm{pc})$ south-east of the optical center. This depression coincides

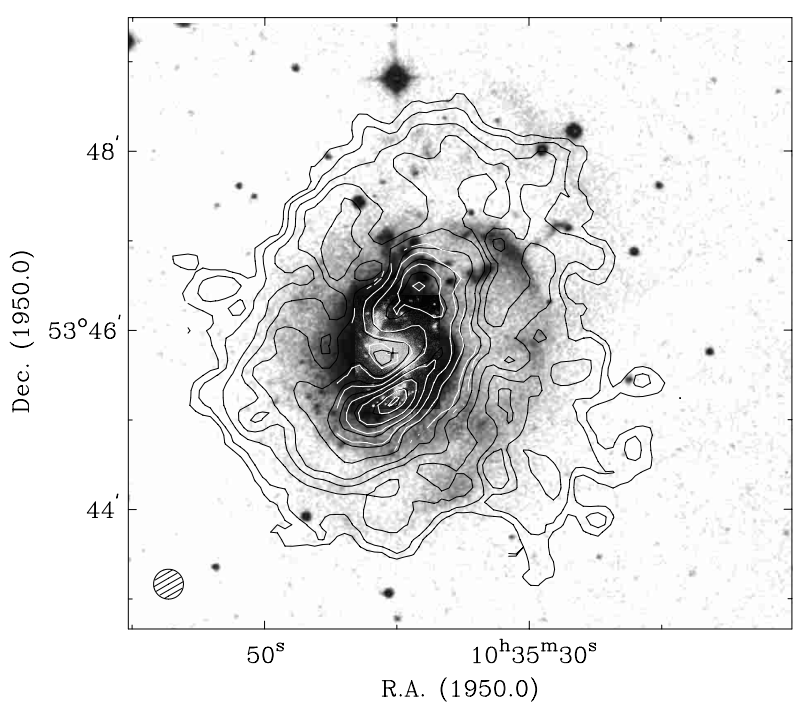

Fig. 8. $20^{\prime \prime}$ H I map (HPBW $=20^{\prime \prime} 0 \times 20$.' 0 ) overlayed on the optical picture (Fig. 1). The contour levels (first:last:increment, in units of $10^{20} \mathrm{~cm}^{-2}$ ) are 1.5, 3:12:3, 18:42:6 and 45 as in Fig. 9c.

with the brightest region of Far UV emission (Smith et al. 1996). The bright inner part is surrounded on all sides by low surface density structures (average densities about $5 M_{\odot} \mathrm{pc}^{-2}$ ). In Fig. 8 this H I map is compared with the optical image. The highest H I density features appear to coincide with the extensions of the optical spiral arms (compare Figs. 8 and 9c). The outer low surface brightness H I seems to coincide only partly with the optical ripple (the "bow") in the north-west and on the southern side. 

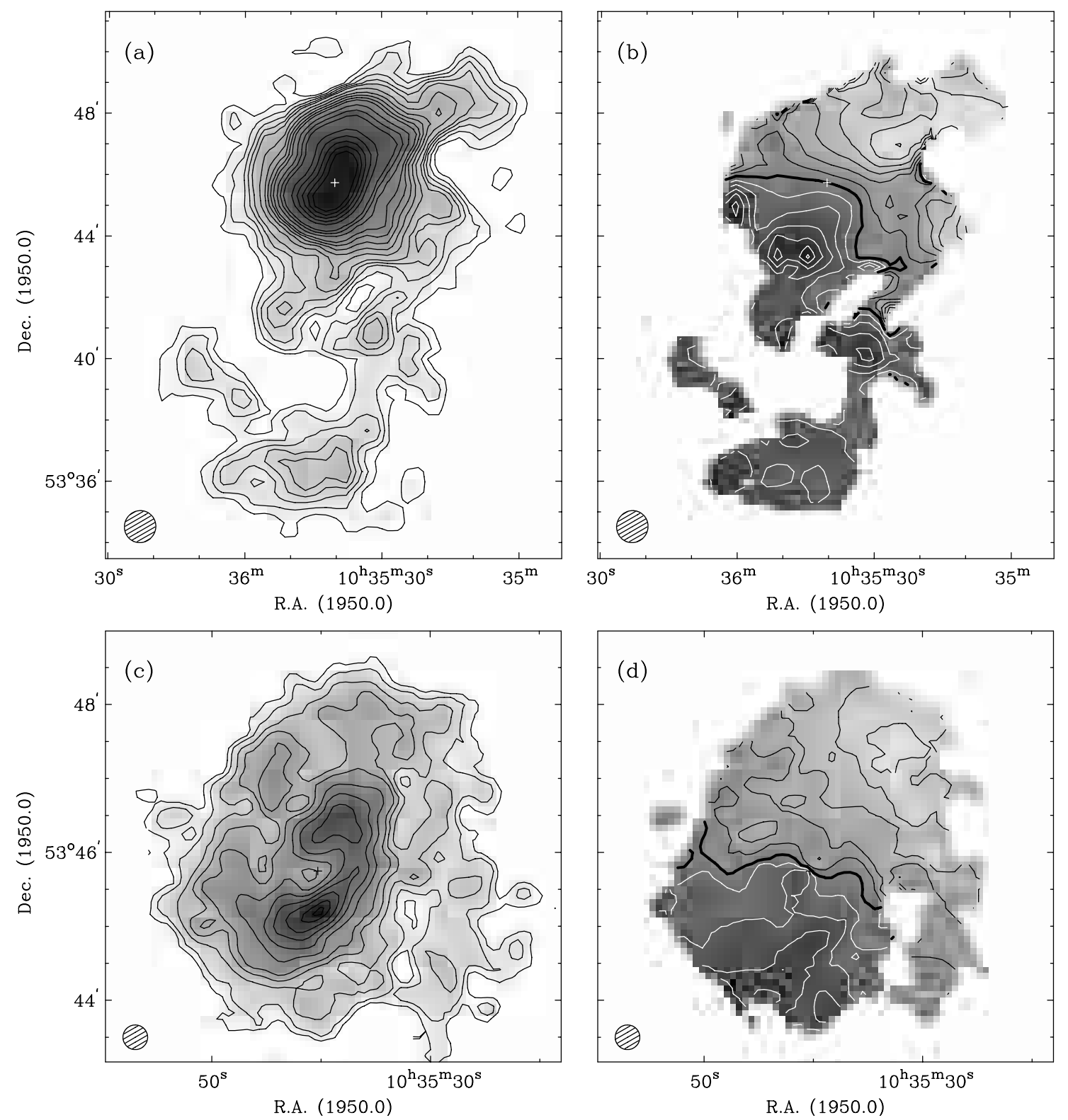

Fig. 9. a) Total H I map $\left(H P B W=60^{\prime \prime} 9 \times 63^{\prime \prime} .0\right)$. The contour levels (first:last:increment, in units of $\left.10^{20} \mathrm{~cm}^{-2}\right)$ are $0.2: 1.4: 0.3$, 2.0:4.4:0.6, 5.6:9.2:1.2 and 11.6:23.6:2.4, b) velocity field $\left(H P B W=60^{\prime \prime} 9 \times 63^{\prime \prime} 0\right)$. The contour levels are in steps of $20 \mathrm{~km} \mathrm{~s}{ }^{-1}$ and the thick contour represents $v_{\text {sys }}$ at $1006 \mathrm{~km} \mathrm{~s}^{-1}$ (the northern side is approaching) c) H I map $\left(H P B W=20{ }^{\prime \prime} 0 \times 20^{\prime \prime} 0\right)$, disk only. The contour levels are (in units of $\left.10^{20} \mathrm{~cm}^{-2}\right)$ 1.5, 3:12:3, 18:42:6 and $45 \mathrm{~d}$ ) velocity field $\left(H P B W=20^{\prime \prime} 0 \times 20^{\prime \prime} 0\right)$. The contour levels are in steps of $20 \mathrm{~km} \mathrm{~s}^{-1}$ and the thick contour represents $v_{\text {sys }}$ at $1006 \mathrm{kms}^{-1}$. The crosses in the panels mark the position of the optical center. The velocity fields are only defined where the surface densities are larger than the values of the second contour in the total Hi maps.

The motion of the $\mathrm{H}$ I disk is clearly dominated by differential rotation although there are irregularities, especially in the outer parts (Figs. 9b and d). At $60^{\prime \prime}$ resolution such irregularities are partly due to the presence of the anomalous $\mathrm{H}$.

The H I velocity dispersion is unusually large (ranging from 20 to $40 \mathrm{~km} \mathrm{~s}^{-1}$ ), especially in the inner highdensity regions which coincide with the stellar disk. H I position-velocity maps taken from the $20^{\prime \prime}$ data at various position angles (see e.g. Fig. 10b) show this very clearly and reveal the presence of faint velocity tails even extending to the quadrant of forbidden velocities (across the systemic velocity line). In the same areas of the disk, H II regions also show large $\left(30-40 \mathrm{~km} \mathrm{~s}^{-1}\right)$ velocity dispersions and large deviations from circular motion (van der Kruit 1976; Grothues \& Schmidt-Kaler 1991; Mulder \& van Driel 1996).

A tilted-ring model (cf. Begeman 1989) was fitted to the velocity field to determine the center of rotation and the inclination angle. This led to an inclination of $56 \pm 7$ degrees for the $\mathrm{HI}$ disk, significantly larger than found for the optical disk (Table 1). 
Table 5. H I and optical parameters for NGC 3310.

\begin{tabular}{|c|c|c|}
\hline Quantity & Units & Value \\
\hline inclination angle (H I) & degrees & $56 \pm 7$ \\
\hline position angle $(\mathrm{H} \mathrm{I})(\mathrm{N} \rightarrow \mathrm{E})$ & degrees & $150 \pm 5$ \\
\hline$v_{\text {sys }}$ (heliocentric) & $\mathrm{km} \mathrm{s}^{-1}$ & $1006 \pm 4$ \\
\hline$M_{\mathrm{HI}}^{\text {disk }}$ & $10^{9} M_{\odot}$ & $2.2 \pm 0.1$ \\
\hline$h_{\mathrm{HI}}$ & $\operatorname{arcsec}$ & $48 \pm 4$ \\
\hline$R_{\mathrm{H} \mathrm{I}}$ & $\operatorname{arcsec}$ & $109 \pm 5$ \\
\hline$R_{\mathrm{T}}^{0} \mathrm{mag}$ & mag & $10.42 \pm 0.07$ \\
\hline$L_{R}{ }^{1}$ & $10^{10} L_{R, \odot}$ & $0.64 \pm 0.04$ \\
\hline$r_{\mathrm{e}}$ & $\operatorname{arcsec}$ & $12 \pm 2$ \\
\hline$\mu_{\mathrm{e}}$ & $R-$ mag $\operatorname{arsec}^{-2}$ & $18.22 \pm 0.05$ \\
\hline$M_{\text {lum }}($ maximum disk $)$ & $10^{10} M_{\odot}$ & 0.2 \\
\hline$M_{\text {tot }}$ (inside $r=110^{\prime \prime}$ ) & $10^{10} M_{\odot}$ & 2.2 \\
\hline$M_{\mathrm{HI}}^{\mathrm{disk}} / L_{R}$ & $M_{\odot} / L_{R, \odot}$ & $0.34 \pm 0.02$ \\
\hline$M_{\mathrm{tot}} / L_{R}$ & $M_{\odot} / L_{R, \odot}$ & 3.4 \\
\hline$M_{\mathrm{H} \mathrm{I}}^{\text {disk }} / M_{\mathrm{lum}}$ & & 1.1 \\
\hline$M_{\mathrm{H} \mathrm{I}}^{\mathrm{disk}} / M_{\mathrm{tot}}$ & & 0.1 \\
\hline
\end{tabular}

1. The $R$-band magnitude is converted to luminosity in solar units using $M_{R, \odot}=4.31$.

The azimuthally-averaged radial density distribution of the H I was calculated from the data at $30^{\prime \prime}$ resolution by averaging the signal in circular rings in the plane of the galaxy. The inclination angle was kept fixed at 56 degrees and the major axis position angle at 150 degrees. The $\mathrm{H} \mathrm{I}$ distribution is approximately exponential. The scalelength $\left(h_{\mathrm{HI}}\right)$ and the radius at a level of $1 M_{\odot} \mathrm{pc}^{-2}\left(R_{\mathrm{H}}\right)$ are given in Table 5. They are similar to those found for galaxies of the same Hubble type (Sbc) as NGC 3310 (Verheijen 1997).

The tilted-ring model fits were also used to derive the rotational velocity. Inclination and position angles were kept fixed. The rotational velocities obtained for an inclination angle of 56 degrees, a position angle of 150 degrees and systemic velocity of $1006 \mathrm{~km} \mathrm{~s}^{-1}$ and estimated for each side separately, are presented in Fig. 10a as dots (approaching side) and crosses (receding side). The $\mathrm{H}$ I rotational velocity is $\sim 60 \mathrm{~km} \mathrm{~s}^{-1}$ at $15^{\prime \prime}$ from the center, just outside the optical ring, and slowly increases further out. Beyond the optical disk, the velocity of the approaching side rises up to $120 \mathrm{~km} \mathrm{~s}^{-1}$ whereas the velocities of the receding side level off at $80 \mathrm{~km} \mathrm{~s}^{-1}$. The curve derived from the $\mathrm{H} \alpha$ observations (van der Kruit 1976) is also shown (rescaled using the $\mathrm{H}$ I inclination). It shows solid body rotation in the inner region up to the starburst ring at $\sim 10^{\prime \prime}$ reaching a maximum of $\sim 105 \mathrm{~km} \mathrm{~s}^{-1}$. This is followed by a sharp drop-off down to $60 \mathrm{~km} \mathrm{~s}^{-1}$ and a slight increase up to $80 \mathrm{~km} \mathrm{~s}^{-1}$ at $70^{\prime \prime}$. The sharp drop in the curve is steeper than Keplerian, indicating that there are non-circular motions present in the inner region (van der Kruit 1976).
Figure 10b shows the rotation curves of the approaching and receding sides superposed (after projection to $v_{\text {rot }} \sin i$ ) on the H I position-velocity map along the major axis. The projected $\mathrm{H} \alpha$ velocities (van der Kruit 1976) are also shown (dotted line). There is good agreement between the $\mathrm{H}$ I and $\mathrm{H} \alpha$ : although the $\mathrm{H}$ I data are too coarse to get a good estimate of the rotation near the center, the lowest contours in the H I position-velocity map show that the $\mathrm{H}$ I motion is consistent with the $\mathrm{H} \alpha$ velocities. Figure $10 \mathrm{~b}$ shows clearly that, in spite of its overall rotation, the $\mathrm{H} \mathrm{I}$ has a peculiar kinematics characterized by a large velocity dispersion and asymmetric profiles. The H I rotation curve derived here has large uncertainties, especially because of the large deviations from circular motion. This uncertainty is of order $20-30 \mathrm{~km} \mathrm{~s}^{-1}$. However, the agreement of the $\mathrm{HI}$ and $\mathrm{H} \alpha$ curves between 20 and 60 arcsec (Fig. 10) indicates that the amplitude of the projected rotation curve adopted here is probably correct. The optical and $\mathrm{HI}$ inclination and position angles (Tables 1 and 5) are also very uncertain and do show large discrepancies.

Because of all these problems and uncertainties the standard analysis with the well-known decomposition in luminous (stars and gas) and dark components (Begeman 1989) may be doubtful. In the following, the results obtained on the mass distribution, the total mass and the mass-to-light ratio should therefore be taken with caution.

The $R$-band surface brightness profile (Fig. 11, dots) was derived from an optical ( $R$-band) image of NGC 3310 (Swaters et al. 2001) using the inclination and position angles as determined from the $\mathrm{H}$ I (Table 5 ). It is best described by an $R^{1 / 4}$ law (Fig. 11, the effective parameters $\mu_{\mathrm{e}}$ and $r_{\mathrm{e}}$ are given in Table 5), except for the inner $10^{\prime \prime}$ (the compact nucleus and the starburst ring). For comparison an exponential disk fit to the bright disk gives a scalelength $h_{R}=9 \pm 1^{\prime \prime}(\sim 0.6 \mathrm{kpc})$ and $\mu_{0}=16.82 \pm 0.05 R$ mag $\operatorname{arcsec}^{-2}$. The surface brightness profile as derived using the inclination and position angle of the optical disk is also shown (circles).

The rotation curve for the disk was calculated from the $R$-band photometric profile (Fig. 11, dots) assuming a truncated disk potential (Casertano 1983) and a mass-tolight ratio constant with radius. The rotation curve of the gas was derived from the $\mathrm{H}$ I density distribution after multiplication by a factor of 1.32 to correct for the presence of helium. The maximal rotation curve of the luminous matter (gas \& stars) is shown in Fig. 10a (dashed curve). A clear discrepancy with the observed rotation curve is visible in the outer parts. Such discrepancies are usually taken to be evidence for the presence of a dark halo. In the inner ring there is a large difference between the maximal rotation curve and the $\mathrm{H} \alpha$ curve. This would imply a mass discrepancy also in the inner region and the presence of a dark mass of about $5 \times 10^{9} M_{\odot}$. However, the sharp drop in the $\mathrm{H} \alpha$ curve is steeper than Keplerian (van der Kruit 1976), indicating that at least part of the discrepancy is caused by non-circular motions.

The mass derived for the maximum disk and the total mass out to $110^{\prime \prime}(=7.1 \mathrm{kpc})$ are given in Table 5 . 

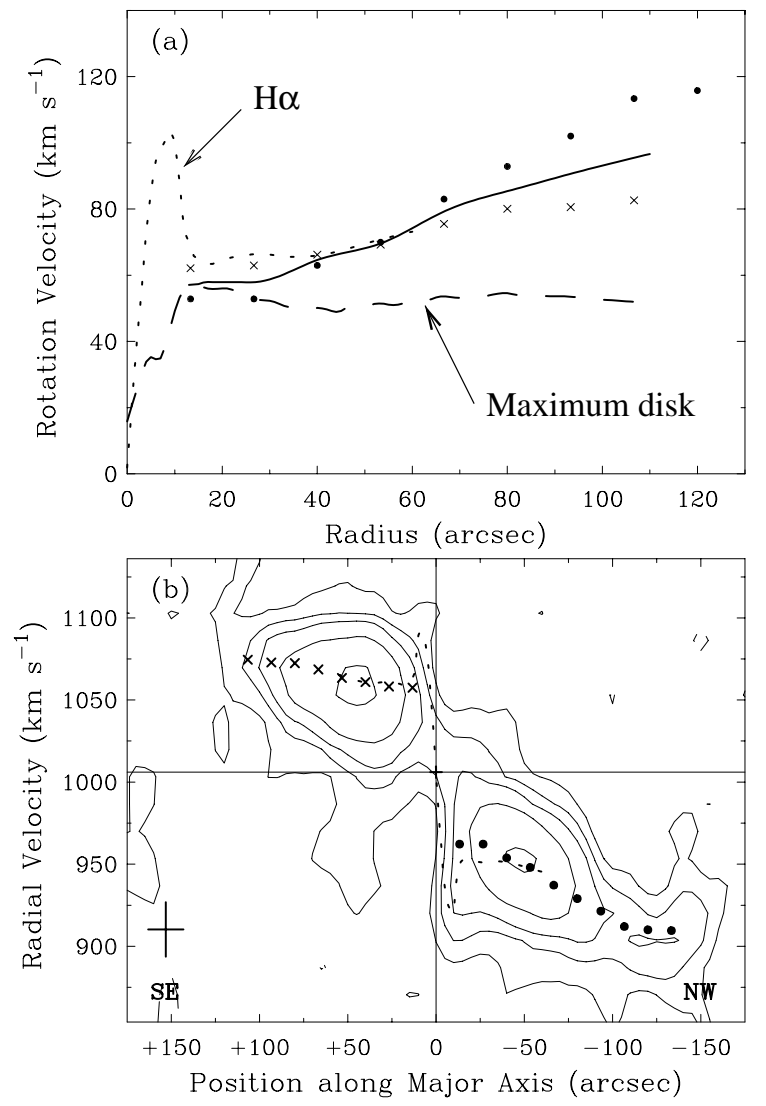

Fig. 10. a) The solid dots and crosses give the H I rotation velocities of the approaching and receding sides respectively, the solid curve shows the mean $\mathrm{H}$ I rotation curve. The dashed curve shows the maximum disk (stars \& gas) rotation curve, the dotted one is that of the $\mathrm{H} \alpha$ obtained by van der Kruit (1976), b) Position-velocity map along the major axis (position angle 150 degrees) at $20^{\prime \prime}$ resolution. Contour levels are -1.7 , 1.7, 3.4, 5.1, 8.5 and $16.9 \mathrm{mJy}_{\text {beam }}{ }^{-1}$. The dots and crosses give the projected $\mathrm{H}$ I rotational velocities for the approaching and receding sides. The dotted curve is from van der Kruit (1976).

When compared to regular spiral galaxies of similar rotational velocity (e.g. Verheijen 1997) NGC 3310 has a rather large $R$-band luminosity (Table 5 ) and low values for the mass-to-light ratio of the maximum disk $\left(M_{\text {lum }} / L_{R}=0.3 M_{\odot} / L_{R, \odot}\right)$ and for the global mass-tolight ratio inside $110^{\prime \prime}\left(M_{\text {tot }} / L_{R}=3.4 M_{\odot} / L_{R, \odot}\right)$. The amplitude of the rotation curve may have been underestimated due to our choice of inclination angle. This may have led to a factor 2 underestimate of the maximum disk and total masses.

\section{Discussion and conclusions}

The Hi observations presented in the previous section have provided new evidence bearing on the dynamics of NGC 3310 and on the origin of the starburst. The main results are the two conspicuous $\mathrm{H}$ I tails connected to the northern and southern sides of the disk and the unusually large velocity dispersion of the $\mathrm{H}$ in the disk.

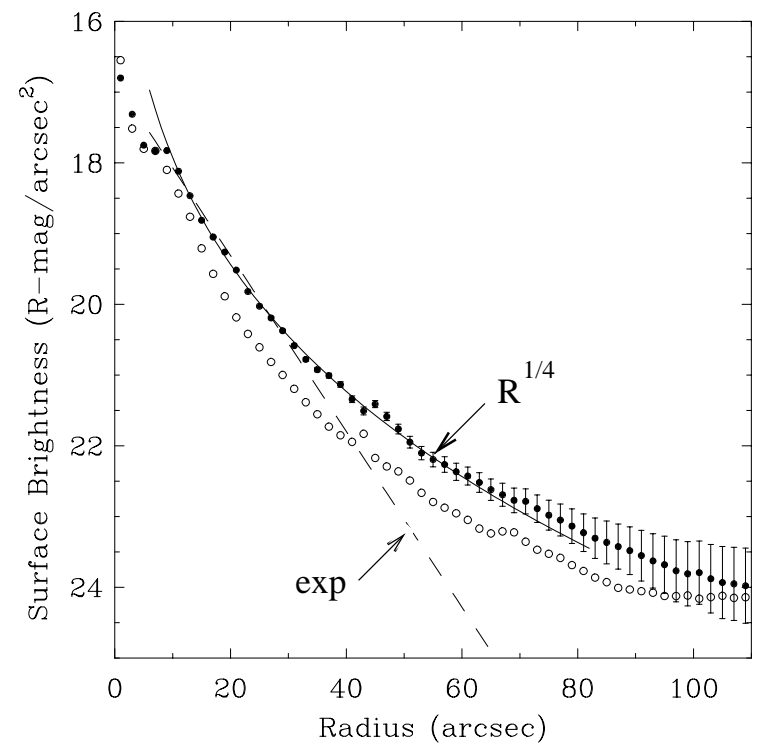

Fig. 11. $R$-band surface brightness profile of NGC 3310. The dots are for the inclination and position angle of the $\mathrm{HI}$ (Table 5), the circles for the inclination and position angle of the $\mathrm{H} \alpha$ (Table 1).

The velocity dispersion of the $\mathrm{HI}$ in the disk, of up to $40 \mathrm{~km} \mathrm{~s}^{-1}$, quite large compared to the values of 7 to $12 \mathrm{~km} \mathrm{~s}^{-1}$ usually found in spiral galaxies, can be attributed for only a small part to profile broadening caused by differential rotation inside the instrumental beam. Most of it must come from an intrinsically high gas turbulence or, more likely, to deviations from circular motion, like the streamings seen in $\mathrm{H} \alpha$. Such deviations would cause, because of the relatively large beam $(20 \operatorname{arcsec}=1.3 \mathrm{kpc})$, broad velocity profiles. The $\mathrm{H}$ I data confirm the results of optical spectroscopy and indicate that the gaseous disk of NGC 3310 is highly disturbed. Large deviations from circular rotation must be present all over the disk and it is not clear whether all non-circular motions are in the plane of the disk.

The two extended tails on opposite sides of the NGC 3310 disk have similar H I masses but different morphologies and kinematics. The one to the south is more extended, reaching out to $51 \mathrm{kpc}$, broader on the sky and more patchy and has almost constant radial velocity (close to the systemic velocity). It has lower H I surface density and no optical counterpart. Its velocity dispersion is around $13 \mathrm{kms}^{-1}$. The tail on the northern side is less extended, half the length, narrower and covers a larger velocity range. Its velocity dispersion is very similar to that of the southern tail.

The northern tail has been constructed with the assumption that the $\mathrm{H}$ I emission with anomalous velocities 804 to $854 \mathrm{~km} \mathrm{~s}^{-1}$ on the northern side of NGC 3310 and the H I coinciding with the "arrow" form one continuous structure. This is clearly in contrast with the interpretation of the arrow as a one-sided jet violently emitted from a compact central source as proposed by Bertola \& Sharp (1984). According to the interpretation favoured here, the 
chain of compact H II regions visible to the north of the bright optical disk would be part of a curved structure continuing to the west with the "arrow" and coinciding with the H I tail. On Plate 2 of Bertola \& Sharp (1984) the continuity between the arrow and the chain of northern optical knots seems obvious. This would imply, however, similar radial velocities of $\mathrm{H} \mathrm{I}$ and $\mathrm{H}$ II, whereas the measurements by Mulder \& van Driel (1996) seem to indicate that the $\mathrm{H}$ II velocities are about $100 \mathrm{~km} \mathrm{~s}^{-1}$ higher. It is possible that there is confusion with $\mathrm{H}$ II emission from the disk. At any rate, it is clear that this point is important for the interpretation proposed here and should be verified by accurately determining the optical radial velocities.

In conclusion, it seems that in all their properties the two H I tails found associated with NGC 3310 resemble the tidal tails seen in gravitationally interacting systems and mergers. It should be noted, however, that in this respect the relative orientation of the two tails may pose a problem (see below).

All the evidence discussed above - the optical ripples, the disturbed kinematics of the gaseous disk and the two H I tails - seems to point to a recent merger event. A merger or a major accretion event for NGC 3310 has been suggested before (Balick \& Heckman 1981; Schweizer \& Seitzer 1988). One possibility is the type of encounter in which an Irr I galaxy is being cannibalized by NGC 3310 as proposed by Balick \& Heckman (1981). Any such explanation should, however, also account for the presence of the two gaseous tails revealed by the present study. The two equal mass, extended tails, the $\mathrm{H}$ I gas content and the small total mass seem to point to a merger between two galaxies of small and comparable masses, of which at least one gas-rich. Indeed, Schweizer's (1978) five characteristics to be expected for a recently merged pair of galaxies - a pair of long tails, an isolated merger-remnant candidate, a single nucleus, chaotic motions and the tails moving in opposite directions - seem to be all present for NGC 3310. For the fifth condition - the two tails moving in opposite directions -, there may be a problem concerning the orientation of the northern tail. It is possible, however, that the tail is not in one plane and that the optical and HI pictures are the projection of a more complex structure. The cluster of northern HII regions does suggest a possible turn of direction from north-north-east to the south-west (see Plate 2 of Bertola \& Sharp 1984). The actual merger process may have been more complex and has, perhaps, involved a third small object as the "bow" and the other ripples seem to suggest. It is remarkable, at any rate, that after such an apparently "major" merger event there should still be a disk. This is clearly different in many respects (in spite of the remarkable $R^{1 / 4}$ photometric profile) from the well studied merger case NGC 7252, the "Atoms-for-Peace" (Hibbard et al. 1994). The unsettled disk we observe now in NGC 3310 could then be either a newly formed disk with spiral arms and star formation going on, or the disturbed disk of one of the progenitors which has survived the merger and is now undergoing new star formation. This would argue in favour of the robustness of disks.

The present observations have added a few more pieces to the interesting puzzle of NGC 3310. It is clear, however, that for a better understanding of the origin of its peculiar morphology and kinematics detailed numerical simulations as done for example by Hibbard \& Mihos (1995) for NGC 7252 are needed.

Acknowledgements. We wish to thank Rob Swaters and Marc Balcells for providing the $R$-band image of NGC 3310, and Tjeerd van Albada and Piet van der Kruit for helpfull comments. We have used the GIPSY package, developed at the Kapteyn Astronomical Institute, for the data reduction and analysis. The Westerbork Synthesis Radio Telescope is operated by the Netherlands Foundation for Research in Astronomy (NFRA/ASTRON), with financial support by the Netherlands Organization for Scientific Research (N.W.O.).

\section{References}

Balick, B., \& Heckman, T. 1981, A\&A, 96, 271

Begeman, K. G. 1989, A\&A, 223, 47

Bertola, F., \& Sharp, N. A. 1984, MNRAS, 207, 47

Broeils, A. H., \& van Woerden, H. 1994, A\&AS, 107, 129

Casertano, S. 1983, MNRAS, 203, 735

de Vaucouleurs, G., de Vaucouleurs, A., Corwin, H. G., et al. 1991, Third Reference Catalogue of Bright Galaxies (New York: Springer)

Duric, N., Seaquist, E. R., Crane, P. C., \& Davis, L. E. 1986, ApJ, 304, 82

Grothues, H. G., \& Schmidt-Kaler, T. 1991, A\&A, 242, 357

Hibbard, J. E., Guhathakurtha, P., van Gorkum, J. H., \& Schweizer, F. 1994, AJ, 107, 67

Hibbard, J. E., \& Mihos, J. C. 1995, AJ, 110, 140

Kikumoto, T., Taniguchi, Y., Suzuki, M., \& Tomisaka, K. 1993, AJ, 106, 466

Lequeux, J. 1971, A\&A, 15, 30

Mulder, P. S., \& van Driel, W. 1996, A\&A, 309, 403

Mulder, P. S., van Driel, W., \& Braine, J. 1995, A\&A, 300, 687

Pastoriza, M. G., Dottori, H. A., Terlevich, E., Terlevich, R., \& Diaz, A. I. 1993, MNRAS, 260, 177

Rhee, M.-H., \& van Albada, T. S. 1996, A\&AS, 115, 407

Schweizer, F. 1978, in Structure and Properties of Nearby Galaxies, ed. E. Berkhuijsen, \& R. Wielebinski (Reidel), 279 Schweizer, F., \& Seitzer, P. 1988, ApJ, 328, 88

Smith, D. A., Neff, S. G., Bothun, G. D., et al. 1996, ApJ, 473, L21

Swaters, R. A., van Albada, T. S., Sancisi, R., \& van der Hulst, J. M. 2001, A\&A, submitted

Telesco, C. M., \& Gatley, I. 1984, ApJ, 284, 557

van der Kruit, P. C. 1976, A\&A, 49, 161

van der Kruit, P. C., \& de Bruyn, A. G. 1976, A\&A, 48, 373

Verheijen, M. A. W. 1997, Ph.D. Thesis, University of Groningen

Walker, M. F., \& Chincarini, G. 1967, ApJ, 147, 416 